\title{
Mesenteric ischemia, new trends, diagnostic methods and algorithms to significantly reduce mortality and morbidity
}

\author{
Balaz P, Rokosny S, Bafrnec J \\ Institute of Clinical and Experimental Medicine, Praha, Czech Republic. jbafrnec@gmail.com
}

\begin{abstract}
This review article summarizes the most recently available data covering the modern trends and methods in the diagnosis and treatment of mesenteric artery disease. Its aim is to point out the critical moments in the whole spectrum of matters such as early diagnosis and treatment of the chronic form of bowel ischemia, role of MDCT in prompt diagnosis, and urgent therapeutic modalities of treating its acute forms. To achieve the best possible results, we must minimize the time of diagnosis while the treatment has to be tailored individually to each patient according to occlusion type, age and co-morbidities (Fig. 2, Ref. 66). Full Text in PDF www.elis.sk. Key words: acute mesenteric ischemia, chronic mesenteric ischemia, multidetector computed tomography, superior mesenteric artery, celiac trunk, great saphenous vein, abdominal aorta, retrograde open mesenteric stent, bypass, endovascular technique, stent, acute abdomen, intestinal fatty acid binding protein, visceral rotation.
\end{abstract}

As apparent from low incidence of mesenteric artery disease, the encounters of vascular surgeons with this disease when compared to those with the diseases of other arteries are very rare. Mesenteric vascular disease may present in its chronic form as chronic mesenteric ischemia (CMI), which in many cases leads to a state of acute mesenteric ischemia (AMI). CMI without therapeutic intervention becomes a great predisposing factor for AMI. Therefore, in the modern world of medicine, the treatment of this rare condition has been assured a rightful place. AMI is already a very serious, life-threatening condition. Little experience of most surgeons leads to many diagnostic errors that crucially extend the time necessary for achieving the definitive diagnosis and providing the emergency treatment. This article reviews major and current information on epidemiology and diagnostic procedures as well as on treatment options for mesenteric ischemia, while unfortunately, eastern medicine has not assured a firm place for any of them.

\section{Epidemiology}

Atherosclerosis (AS) of mesenteric arteries is often manifested in patients with generalized AS. The incidence increases with age while the prevalence among the population over 65 years in Europe and North America is approximately $20 \%$ (1). Autopsy studies have demonstrated that stenosis of over $50 \%$ stenosis of at least one of the mesenteric arteries occurs in 6-10\% of population (2). Asymptomatic stenosis of over $50 \%$ afflicting the SMA or celiac trunk (TC) is present in $27 \%$ of patients undergoing

Institute of Clinical and Experimental Medicine, Praha, Czech Republic

Address for correspondence: J. Bafrnec, MD, Institute of Clinical and Experimental Medicine, Videnska 1958/9, CZ-140 21 Praha 4, Praha, Czech Republic.

Phone: +420.608177328 angiography for peripheral vascular disease (PVD) (3). Patients undergoing aortofemoral bypass surgery for occlusive disease of the pelvic arteries are affected by concomitant mesenteric artery disease in approximately $70 \%$ (4). Endean confirmed that over $20 \%$ of patients with AMI, had clinical signs of CMI (5). Thomas described in his study that up to $86 \%$ of patients with AS changes in all three visceral arteries had clinical manifestations of CMI or died of AMI in median follow up 2.6 years (6).

Patients with AS of mesenteric arteries have significantly enlarged collaterals between the celiac trunk (CT), superior mesenteric artery (SMA), inferior mesenteric artery (AMI), and internal iliac artery (IIA). That is why an affliction of only one of these main arteries supplying the small intestine remains largely free of symptoms (7). Chronic mesenteric ischemia (CMI) may stay asymptomatic for months to years.

In a study based on autopsy or surgery, Acosta described the incidence of AMI in the Swedish population in 1970-1982 as $12.9 / 100,000$ inhabitants/year (8). Of the total of 402 patients, 270 $(67.2 \%)$ were confirmed to suffer from acute occlusion of SMA, 63 (15.7\%) had mesenteric venous thrombosis (MVT), 62 (15.4 $\%$ ) developed nonocclusive mesenteric ischemia (NOMI), and in $7(1.7 \%)$, the etiology was not found. The overall incidence of acute SMA occlusion described by Acosta in the same period was 8.6/100.000 patients/year. It is interesting that the acute closure of SMA is more frequent in this group than ruptured abdominal aortic aneurysm (RAAA). The incidence of SMA occlusion is increasing with age and is more common among women (9). In his published study, Acosta proved SMA occlusion mortality during 1970-1982 to be $6 / 1000$ deaths and hospital mortality to be $93 \%(10)$. According to the same author, in 2004-2009, the mortality has been reduced to $58 \%$ and hospital mortality came down to $36 \%$. This significant decrease is explained by bringing out a better diagnostic approach, and especially by introducing the multidetector CT angiography (CTA). 
In a study of 213 cadavers with acute closure, the ratio of SMA embolism versus thrombus was 1:4. Thrombotic occlusion was located more proximally than the embolic occlusion; infarction of the gut was more extensive and associated with thrombosis of AA and disseminated carcinoma (11). Thrombosis or severe AS changes in the celiac trunk were found in $33 \%$ of patients with thrombosis of SMA, while more than $73 \%$ of patients with acute SMA thrombosis had signs of chronic intestinal ischemia (12).

The results of epidemiological studies have shown that 1) acute mesenteric ischemia is very often associated with acute abdominal pain 2) the source of acute SMA embolism is often in the heart while at the same time, embolism often affects other visceral arteries 3 ) the presence of CMI requires a prompt solution because the risk of AMI accompanied by great mortality is significantly high (9).

\section{Acute mesenteric ischemia}

In 1951, Klass was the first surgeon to perform embolectomy of SMA due to its acute occlusion (13). Unfortunately, despite the fact that nearly 60 years have passed since the first acute revascularization of SMA, the mortality caused by AMI remains very high, ranging between $60-80 \%(14,15)$. According to Acosta, if the reperfusion of the bowel is achieved in less than 12 hours from acute occlusion, the mortality can be reduced to $25 \%$; if in less than 24 hours then to $50 \%$ (8). The reason for such high mortality lies in the fact that only one third of patients with AMI were diagnosed and treated properly $(10,16)$. In $40-50 \%$, the main cause of AMI is arterial embolisation from the heart, mostly affecting SMA due to its small angle of branching from the aorta $(17,11)$. Acute mesenteric thrombosis on pre-existing AS changes was found in $25-30 \%$ of all cases (18).

\section{Diagnosis of AMI}

Early diagnosis of AMI is the only chance for success in this life-threatening condition. If the diagnosis is imprecise or delayed, it leads to almost $100 \%$ mortality. The diagnosis of AMI is extremely difficult because the course of the disease is often atypical, especially in old ventilated patients with co-morbidities and in many cases after major cardiac surgery. Proper diagnosis is based on clinical symptoms dominated by sudden (but not disastrous) diffuse pain in the epigastrium, as well as by fast emerging fatigue and overall deterioration. Frequent cramps are accompanied by diarrhea, usually without vomiting. Unfortunately these symptoms are also typical for other acute abdomen conditions (AA) as pancreatitis, cholecystitis, appendicitis and ileus. AMI is therefore easily misdiagnosed. Objective physical findings are therefore nonspecific. This stage is replaced after approximately 6 hours with relative subjective improvement and development of paralytic ileus. The second phase of the illness lasts for 6-12 hours. The last stage, after 12 hours, is dominated by the typical picture of peritonitis (19).

The laboratory results show typical but nonspecific increases in leukocytosis, CRP or procalcitonin, and therefore the elevation of inflammatory parameters cannot determine the diagnosis of AMI. Serum markers for the diagnosis of AMI are completely insensitive and nonspecific (20). A promising diagnostic tool appears to be immunologically determined "intestinal fatty acid-binding protein". Its elevation was detected in intestinal infarction. Routine testing to establish the diagnosis is still limited (21). Some studies have shown promising results with the use of high levels of serum D-dimmer in the context of history and clinical condition in the diagnosis of AMI $(22,23)$.

In $25 \%$ of patients with AMI, the routinely used imaging techniques such as X-ray of the abdomen are without pathology (24). Ileus may already be an advanced finding of intestinal ischemia. Therefore, this method is nonspecific for AMI.

An extended use of ultrafast multidetector computed tomography angiography (MDCT) has been assured a stable place in the diagnosis of AMI and CMI (25). In addition to the diagnosis of stenosis or occlusive vascular disease, CT scan can exclude or further confirm the possible pathology present in the abdominal cavity. Proper timing of the administration of contrast medium and ultrathin sections provide the clinician with an excellent picture of visceral arteries $(26,27)$. One of the most important moments for proper implementation of MDCT is the exact timing of administration of contrast medium $(\mathrm{CM})$ which has to be synchronized with the CT scans. The use of traditional CM orally is currently not recommended due to the production of artifacts. According to the protocol, it is recommended to administer "negative" CM orally (500-700 ml water), immediately before testing. Promptly indicated computed tomography can provide important information about visceral arteries, and thus significantly facilitates the following clinical decisions.

Traditional multiplanar angiography is the most accurate diagnostic examination of both AMI and CMI. In addition to accurate diagnosis, in some cases it provides the possibility of fast treatment with endovascular techniques (local administration of vasodilators, balloon angioplasty, stenting, thrombolysis and aspiration thrombectomy) $(28,29)$. Per-operative angiography is establishing its position in a hybrid approach to the treatment of AMI.

Duplex ultrasonography (USG) is a noninvasive examination, which may be useful for identifying significant stenosis of the celiac trunk and SMA. Ultrasound is used only in cases of CMI for assessing the intestinal peristaltic movement, and for excluding other pathology in differential diagnosis of AMI (30).

Magnetic resonance imaging (MRI) is currently rarely used in the diagnosis of AMI, because it is time-consuming and needs sophisticated software as well as sufficient erudition of radiologists. MRI tends to overestimate the degree of stenosis (31), and the evaluation of secondary changes such as induration of intra abdominal fat as well as of bowel wall thickening is more problematic than when scanned by CT (19).

The importance of diagnostic laparoscopy in AMI is controversial and limited. This method is not suitable for objective evaluation of the color of serous membranes, assessment of bowel peristalsis, and especially for investigation of all parts of the intestine. It may increase the risk of false negative results. Therefore, this method is not widely acceptable for the diagnosis of AMI (32).

\section{Treatment of AMI}

Surgical revision is indicated in all patients with the signs of acute abdomen based either on AMI or other etiologies. The pa- 
tient including his/her lower extremities is covered by surgical sheets to the knees to retain the possibility of dissecting the great saphenous vein (GSV) or femoral vein (VF). The first step after laparotomy ranging from the xyphoid process to the symphysis, is the assessment of ischemia by evaluating the viability of intestinal loops, mainly in patients in whom angiography was performed preoperatively. An emphasis should be paid on visual comparison of potentially viable bowel. Intestinal necrosis is characterized by ash-gray color lacking the reflective shining. It is important to realize that a bowel showing the signs of ischemia can be vital after revascularization. Peristalsis is mostly absent, but it clearly does not exclude the presence of ischemia. Next step is either bowel resection with subsequent revascularization or vice versa. The first method has an advantage of eliminating the danger of perforation and subsequent contamination of the abdominal cavity by stercoral content with the potential development of peritonitis. On the other hand, after bowel resection prior to revascularization, we are not able to confidently assess the degree of vitality of the loops. The decision to perform resection and revascularization procedure depends on the habits and experience of the surgeon.

\section{Superior mesenteric artery embolectomy}

The dissection of SMA is done by retracting the omentum and colon transversum cranially while the small intestine is to be retracted caudally. Peritoneal incision is horizontal on the base of mesocolon transversum while revealing the SMA. Careful and precise dissection of the mesentery is crucial to avoid injury of the superior mesenteric vein, which is topographically to the left and caudal to SMA. We must also lay emphasis on the branching of jejunal arteries along with medial colic artery. After exposing SMA and total heparinisation, we perform either a transverse or longitudinal arteriotomy. After transverse arteriotomy followed by successful embolectomy, we can directly suture the artery. However, if the conditions require bypass surgery, the transverse incision is inadequate. Therefore most surgeons perform a longitudinal arteriotomy, which can be closed with continued stitch or venous patch from the great saphenous vein (GSV). Proximal embolectomy is performed with Fogarty 3-4 F catheter until the inflow is renewed. For distal embolectomy we use $2-3 \mathrm{~F}$ catheter in order to avoid the possibility of causing either a dissection or perforation resulting in bleeding into the mesentery. After successful embolectomy it is recommended to infuse small quantities of recombinant tissue plasminogen activator (rt-PA) to the distal SMA (19).

\section{Mesenteric bypass}

In cases of SMA closure due to atherosclerosis, the performance of thrombectomy/embolectomy alone does not ensure the adequate blood flow. Therefore the method of choice is that of revascularization bypass surgery. In AMI we focus on renewal of blood flow only in SMA. In this case, the recommendations to revascularise two arteries as in CMI are not justified (19). The first issue is the choice of vascular prostheses. Unlike in CMI, where the method of choice is a synthetic vascular graft, in cases of AMI presenting with necrosis of intestinal loops with possible perforation, the use of prosthesis made of synthetic material incurs high risk of potentially inducing infection with catastrophic consequences. Therefore, for mesenteric bypass in AMI, the method of choice is to use autologous material (GSV, VF). For the reasons above, it is necessary to have the patient well prepared for harvesting a lower extremity venous graft. When comparing GSV to VF, the latter has better results (33). In practice however, the time to dissect either VF or GSV is limited, wherefore synthetic prosthesis bypass is often used (19). When using the synthetic prostheses (Dacron, PTFE reinforcement, 6-8 mm), it is necessary to pay attention to prevention of infection. Kazmer described the technique of wrapping the prosthesis in an omental flap, which significantly reduces the risk of infection (34). The orientation of mesenteric bypass may be ambivalent, antegrade or retrograde. Most surgeons use the retrograde bypass techniques based on placing the central anastomosis at common iliac artery (AIC), right/left while the bypass has a configuration of a "lazy" $\mathrm{C}$. This technique eliminates the clamp on the aorta and prevents the bypass from "kinking". If the infrarenal aorta is sclerotic or with an aneurysm, the antegrade aorto-mesenteric bypass is indicated, even though it brings about a high risk of kinking. The advantage of antegrade bypass is in the fact that the suprarenal aorta is often of good quality and the orientation of bypass is more physiological with low risk of kinking. On the other hand, the preparation is technically demanding, and the suture causes hemodynamic stress during aortic clamping (19).

\section{Endovascular techniques}

Continuous improvements to endovascular techniques have made this option of AMI treatment more accessible and widespread. Advanced hybrid operating rooms and combined approaches to endovascular treatment with open surgery is the most modern method of treatment of AMI (35). Although endovascular methods offer several different therapeutic approaches, open surgery remains the first choice in the treatment of AMI. One of the endovascular options is aspiration thrombectomy of SMA emboli. Several articles have been published but groups of patients are small and with inconclusive results $(36,37,38)$. Studies on thrombolytic therapy (TL) of acute occlusion of SMA are scarce in literature (39). Wide use of TL is not common for two reasons. The first is that the time required for the effect of thrombolysis is too long for a patient suffering from developed acute intestinal ischemia. The second reason is the high risk of bleeding during bowel necrosis already emerging (35). Percutaneous angioplasty can be performed during diagnostic angiography, after TL or after aspiration thrombectomy.

In case of AMI with signs of acute abdomen, exploratory laparotomy is always indicated. Revascularization can be done by surgery or percutaneous endovascular approach through the common femoral artery (AFC) or brachial artery (AB). After successful endovascular treatment, the exploratory laparotomy is indicated in accord with the clinical condition of patient. The diagnostic procedure and indications arousing suspicion of AMI are shown in the scheme below (Fig. 1).

As previously mentioned, endovascular angioplasty is not the first choice in the treatment of AMI. It requires an experienced radiologist; it is time-consuming and has only a limited possibil- 


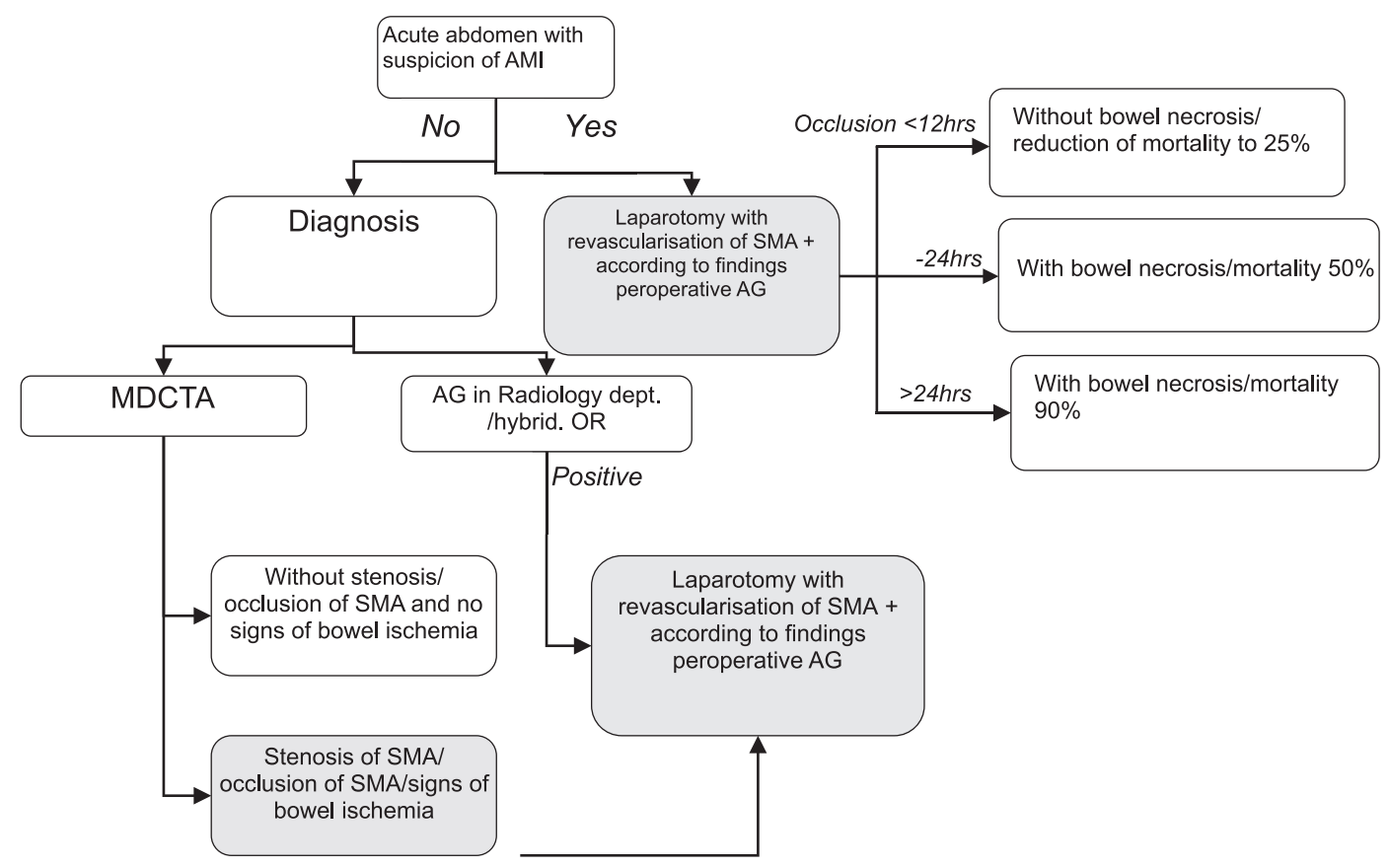

Fig. 1. Acute occlusive mesenteric ischemia-initial clinical evaluation/resuscitation. IR - interventional radiology, MDCTA - multidetector CT arteriogram.

ity of evaluating the bowel viability. Finally more experience has yet to be documented in publications. One way to combine the endovascular and open surgery approaches is that of retrograde open mesenteric stent (ROMS). Wyers published a method of stenting the SMA after thrombectomy during an open surgery in patients with AMI (19). Although the group had only 6 patients, the technical success was $100 \%$. This method is recommended by Wyers for further testing for it to become an alternative attractive treatment option for AMI.

\section{Chronic mesenteric ischemia}

Chronic mesenteric ischemia, type of arterial insufficiency, was first described in 1869 by Chienne (41). Mayard Shaw described the first surgical endarterectomy (EA) of SMA, which led to relief from symptoms associated with CMI (42). Unlike AMI, CMI is not an acute life-threatening condition. This diagnosis can be sometimes discovered only by a specific imaging examination without the supporting clinical symptoms. On the other hand, it is an important predisposing factor for the development of fatal AMI $(43,44)$. In the U.S., the incidence of CMI is reported to be less than 1 case/100,000 hospital admissions and less than $2 \%$ of total admissions for gastrointestinal problems (45). According to the U.S. National Registry, in 2000-2006 there were 5,583 patients treated for the diagnosis of CMI (46). The pathophysiology of CMI symptoms is based on an inadequate blood flow to the mesenteric circulation due to increased metabolic needs during digestion. Intestinal circulation (TC, SMA, and IMA) requires $10-20 \%$ of basic cardiac output and about $35 \%$ of postprandial cardiac output, where $70 \%$ of the circulation supplies the metabolic needs of the intestinal mucosa (47). Depending on the composition of digested food, the increase in intestinal blood circulation begins 30-60 minutes after eating and lasts for 2-6 hours (48). CMI symptoms usually appear when two or all three major mesenteric arteries are affected, or when previous surgery has interrupted the connections between the anatomical collaterals. When there is an isolated but significant stenosis of one of the three arteries while the collateral circulation is intact, the clinical symptoms may be often absent. In addition to typical symptoms of CMI we can also observe the so-called "relative steal" which brings about abdominal pain before the food reaches the small intestine. This situation occurs when TC and SMA are afflicted with stenosis of over $50 \%$. The blood flows from the small intestine via the collateral circulation to the stomach and the small intestine remains ischemic (49). Although AS of visceral arteries is the leading cause of CMI, this diagnosis can be also caused by vasculitis, fibromuscular dysplasia, neurofibromatosis, dissection of arterial walls, trauma, embolism, aortic coarctation and Dunbar syndrome (celiac trunk compression syndrome).

\section{Diagnosis of CMI}

Patients with this diagnosis are usually $70-80$ years old, predominantly women. A typical symptom of CMI is postprandial pain and weight loss of $10-15 \mathrm{~kg}$. The pain is mainly associated with food intake, which traditionally begins 15-30 minutes after eating and lasts for 5-6 hours until the food is digested. The pain is typically deep and colic-like. It must be distinguished from peritoneal signs of intestinal infarction, gastrointestinal perforation or ulcer disease. The patient history often reveals smoking and systemic signs of AS (50). The first step in the diagnosis of CMI is the exclusion of malignancy and secondly the use of imaging methods to 


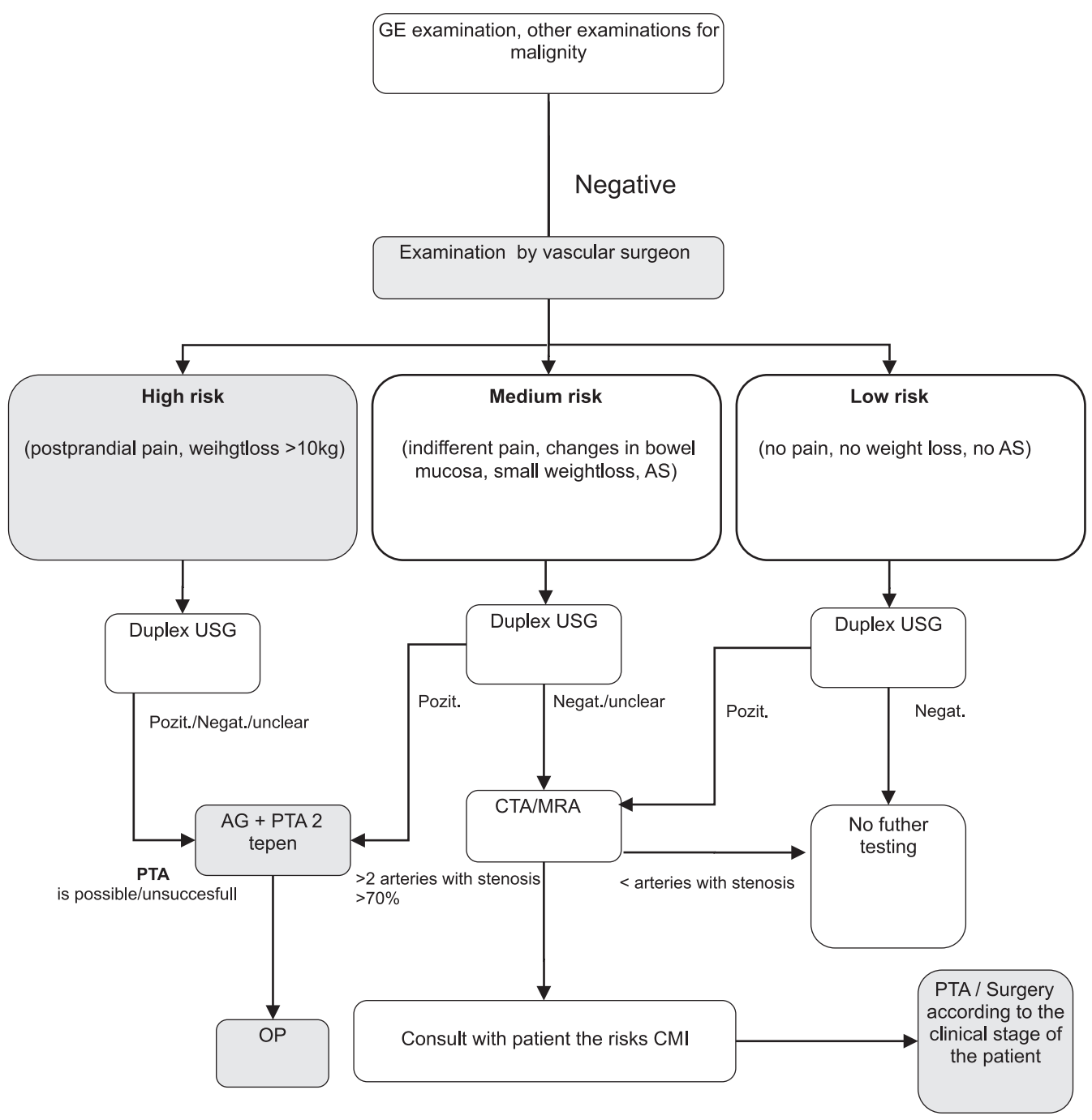

Fig. 2. Diagnostic algorithm in suspicion of CMI. AS - atherosclerosis, GE - gastroenterological.

diagnose the involvement of visceral arteries. The routinely used methods include duplex ultrasonography, CTA and MRA but the gold standard for diagnosis of visceral artery involvement remains to be that of conventional angiography. The various imaging methods are described in the diagnosis of AMI. The diagnostic process followed by therapeutic options is shown in Figure 2.

\section{Surgical treatment of CMI}

CMI is often associated with atherosclerosis of at least two from three major mesenteric arteries. The aim of treatment is to relieve the patient from symptoms, normalize the weight and especially to provide prophylaxis of AMI. Surgery is indicated in asymptomatic patients undergoing aortic reconstruction with critical lesions on all three major mesenteric arteries, as well as in symptomatic patients where the risk of AMI is involved in $43 \%$ of these patients (51). Open revascularization provides immediate relief of symptoms in most patients, even though it has higher mortality risk compared to endovascular techniques
(15\% to $8 \%$ ) (52). Mesenteric bypass is used more often in patients where PTA was not technically feasible or failed, in patients with occlusion of mesenteric stent and in-stent stenosis when re-intervention failed. Open revascularization is also recommended for all other causes of non-atherosclerotic CMI (52). Surgical revascularization is performed by means of antegrade or retrograde type of bypass on one or more mesenteric arteries, with inflow from the abdominal aorta (AA) or pelvic arteries. Older, cachectic patients with co-morbidities such as low ejection fraction (EF), pulmonary and renal dysfunction, atherosclerotic AA are usually not able to bear aortic cross-clamping. In such cases, the pelvic artery serves as an alternative inflow artery, ensuring sufficient flow associated with a significantly lower risk for the patient (52). The method of retrograde open mesenteric stent (ROMS) described previously in the treatment of AMI, is among options also in the treatment of CMI in high risk patients or to prevent graft infection in patients with contamination of the peritoneal cavity. There is little experience with this method but it is promising. 
Supraceliac antegrade AA-TC and SMA bypass

This type of reconstruction is indicated for low-risk patients with lesions in two mesenteric arteries, where the supraceliac aorta is without significant calcification. The preferential conduit is that of knitted bifurcation prosthesis. Transperitoneal median laparotomy or bilateral sub-costal laparotomy is followed by inspection of abdominal cavity in order to exclude malignancy. The operation continues by opening the small omentum, cutting the left triangular ligament and releasing the left lobe of the liver. Esophagus with established nasogastric tube (NGT) is pulled to the left while the stomach is pulled slightly caudally. After dissecting the diaphragmatic crura, the supraceliac AA is stripped. It is usually sufficient to dissect the celiac trunk, proximal parts of hepatic and splenic artery. The left gastric artery is often thin and can be safely interrupted, thereby facilitating the anastomosis to the trunk and tunneling the graft to SMA behind the pancreas. The transverse mesocolon is pulled cranially, and at the base of mesentery, SMA is isolated. After full heparinisation, mannitol-induced diuresis, we proceed with the clamping of AA, vertical or slightly arched arteriotomy and central anastomosis end-to-side (EtS) with 14x7 mm knitted prosthesis.

This should not exceed 20 minutes. The left arm is then stretched behind the pancreas, and subsequently the EtS anastomosis on SMA is performed (graft length must be measured while releasing the retractor in order to prevent the kinking of bypass). Anastomosis on TC is usually an end-to-end or on the hepatic artery EtS (52).

\section{Iliac or infrarenal aortic retrograde mesenteric bypass}

In case of technical limitations or high risk of supraceliac bypass, the infrarenal aorta or one of the iliac arteries can be used as an inflow artery. The best place to start central anastomosis is the right iliac artery (RIA) while braided $8-10 \mathrm{~mm}$ bifurcation prosthesis is used as a conduit. The technique is identical to the operation described above in the treatment of AMI (19). According to the current study, the results of retrograde reconstruction are the same as those of antegrade reconstruction $(53,54)$.

\section{Endarterectomy}

Transaortic endarterectomy is indicated for exceptional cases but can be successfully used in patients who are not candidates for endovascular treatment, have contaminated peritoneal cavity or gastrointestinal perforation, as well as those with significant obesity or history of radiation therapy. The surgical mortality at the University of Wisconsin is excellent, namely $3.8 \%$ of 80 patients (55). The surgical approach is that of median laparotomy with medial visceral rotation, which after total heparinisation allows the performance of transaortic endarterectomy of celiac trunk and SMA.

Surgical treatment of CMI is a technically demanding and risky procedure, but with proper indication, patient selection and good technique, it has excellent results. Perioperative mortality is lower than $3 \%(55,56,57)$. After the surgical treatment, the improvement from CMI symptoms is observed in $77-100 \%$. The recurrence of symptoms within 5 -year follow up was observed in $12 \%$ of patients on average (58). When compared with angioplasty, the comparative analysis shows a lower rate of restenosis and reintervention (58). Regarding the target artery for revascularization, most surgeons believe that for the treatment of CMI, it is sufficient to use SMA as the only revascularised artery. An Oregon group has published excellent results based on using single artery revascularization (59). On the other hand, Park et al. recommends revascularization of two arteries when possible, i.e. in low-risk patients (60).

\section{Endovascular treatment of CMI}

Endovascular treatment of CMI has become the first choice of treatment in recent years because of its low invasiveness as well as due to low mortality and morbidity compared with open revascularization. It is the first choice in patients with CMI, while open surgical revascularization is the method of first choice for AMI. At the Mayo Clinic during the years 1990-2009 there were performed 327 revascularizations in patients with CMI. More than $70 \%$ of them were performed with the endovascular approach. Stent insertion for short $(<2 \mathrm{~cm})$ mesenteric artery stenosis with minimal or moderate calcification is a very successful treatment method. With heavy calcification, occlusion, extensive stenosis and small diameter arteries, angioplasty is associated with a high risk of distal embolisation, re-stenosis, and reintervention. Although these lesions are usually not a hindrance for endovascular approach, Oderich et al. recommended open surgery for patients with low perioperative risk or anatomy unsuitable for endovascular revascularization $(61,62)$. The endovascular technique uses primary stenting of mesenteric arteries through the femoral approach. Failure of endovascular techniques is between $5-10 \%$, and 30 -day mortality is $0-16 \%$ according to various studies, but in most studies it is lower than $5 \%$. Perisurgical mortality in the multicenter study published by Schermerhorn (63) was 3.7\% and the primary patency at 12 months was $65-85 \%(64,65)$.

After summarizing the available results in the treatment of CMI, it can be said that currently more than $70 \%$ of patients were treated with endovascular approach. This treatment option is preferred in high-risk group of patients but also in patients with low risk and well-situated lesions. Open revascularization has been assured an important place in cases of localized lesions, anatomically unsuitable for PTA, extensive stenoses, occlusions, tandem lesions, small arteries and occluded stents.

\section{Nonocclusive mesenteric ischemia (NOMI)}

Although this entity is not a part of the concept of this review, it is important to mention it. In reality, we can encounter critically ill patients showing signs of intestinal ischemia despite having all 3 mesenteric arteries with no signs of occlusion, embolism, thrombosis, dissection or strangulation. This condition is known as NOMI. A typical patient is that of advanced age with heart disease, or sepsis, and inotropic support. The overall incidence verified at autopsies or during surgery in the Acosta study was 2.0/100,000 inhabitants/year (63). An important clinical fact is that up to 40 $\%$ of patients with SMA stenosis had a potentially curable disease with PTA. Publications about NOMI are insufficient, mostly reviews or work studies concerning a small group of patients. Diagnosis of NOMI is difficult and with the introduction of MDCT, four criteria for early diagnosis were introduced as follows: (1) symptoms of ileus, abdominal discomfort and abdominal pain (2) 
inotropic support (3) episode of hypotension (4) slow elevation of transaminases. If three out of the four criteria have been met in a patient after cardiac surgery, or in a patient on dialysis, the indication for early MDCT is made. Pharmacological treatment consists of high doses of intravenous prostaglandin E1, papaverine as vasodilators, nitroglycerin, or glucagon and antibiotics (66).

\section{Conclusion}

Mesenteric artery disease, especially in form of chronic and acute mesenteric ischemia, is a very serious condition with an increasing incidence in the western world. Even with the most modern diagnostic measures and treating options, it remains a challenge for specialists in emergency and operating rooms. However, recent studies suggest that with good cooperation and prompt intervention, we are able to significantly reduce the mortality in acute mesenteric ischemia. This summary of the most up-to-date data also shows that generalized atherosclerosis, quietly affecting the mesenteric arteries, has nonspecific symptoms and is a great predisposing factor for acute occlusion. Therefore, the diagnosis and treatment of CMI are becoming powerful tools in preventing and reducing the incidence of AMI. The authors hope that this review article is going to serve as a good source of most recent information, and has the potential to make this topic more popular among a broad spectrum of specialists.

\section{References}

1. Roobottom CA, Dubbins PA. Significant disease of the celiac and superior mesenteric arteries in asymptomatic patients; Predictive value of Doppler sonography. Am J Roentgenol 1993; 161: 985-988.

2. Li KC, Hopkins KL, Dalman RL, Song CK. Simultaneous measurement of flow in the superior mesenteric vein and artery with cine phasecontrast MR imaging; Value in diagnosis of chronic mesenteric ischemia; Work in progress. Radiology 1995; 94: 327-330.

3. Valentine RJ, Martin JD, Myers SI, Rossi MB, Clagett GP. Asymptomatic celiac and superior mesenteric artery stenoses are more prevalent among patients with unsuspected renal artery stenoses. JVasc Surg 1991; 14: 195-199.

4. Bradbury AW, Brittenden J, McBride K, Ruckley CV. Mesenteric ischaemia; A multidisciplinary approach. Br J Surg 1995; 82: 1446-1459.

5. Endean ED, Barnes SL, Kwolek CJ, Minion DJ, Schwarcz TH, Mentzer Jr. RM.Surgical management of thrombotic acute intestinal ischemia. Ann Surg 2001; 233: 801-808.

6. ThomasJH,BlakeK,PierceGE,HermreckAS, SeigelE. The clinical course of asymptomatic mesenteric arterial stenosis. J Vasc Surg 1998; 27: 840-844.

7. Brandt LJ, Boley SJ. AGA technical review on intestinal ischemia: American Gastrointestinal Association. Gastroenterology 2000; 118: 954-968.

8. Acosta S, Wadman M, Syk I, Elmståhl S, Ekberg O. Epidemiology and prognostic factors in acute superior mesenteric artery occlusion; A population-based study. J Gastrointest Surg, in press.

9. Acosta S. Epidemiology of Mesenteric Vascular Disease; Clinical Implications. Semin Vasc Surg 2010; 23 (1): 4-8.

10. Acosta S, Ögren M, Sternby NH, Bergqvist D, Björck M. Incidence of acute thromboembolic occlusion of the superior mesenteric artery; A population-based study. Eur J Vasc Endovasc Surg 2004; 27: 145-150.
11. Acosta S, Ögren M, Sternby NH, Bergqvist D, Björck M. Clinical implications for the management of acute thromboembolic occlusion of the superior mesenteric artery; Autopsy findings in 213 patients. Ann Surg 2205; 241: 516-522.

12. Acosta S, Sonesson B, Resch T. Endovascular therapeutic approaches for acute superior mesenteric artery occlusion. Cardiovasc Intervent Radiol 2009; 32: 896-905.

13. Klass AA. Embolectomy in acute mesenteric occlusion. Ann Surg 1951; 134: 913-917.

14. Kassahun WT, Schulz T, Richter O, Hauss J. Unchanged high mortality rates from acute occlusive intestinal ischemia; Six year review. Langenbecks Arch Surg 2008; 393: 163-171.

15. Oldenburg WA, Lau LL, Rodenberg TJ, Edmonds HJ, Burger CD. Acute mesenteric ischemia; A clinical review. Arch Intern Med 2004; 164: 1054-1062.

16. Mamode N, Pickford I, Leiberman P. Failure to improve outcome in acute mesenteric ischemia; Seven-year review. Eur J Surg 1999; 165: 203-208.

17. Lock G. Acute intestinal ischaemia. Best Pract Res Clin Gastroenterol 2001; 15: 83-98.

18. Mansour MA. Management of acute mesenteric ischemia. Arch Surg 1999; 134: 328-330.

19. Wyers MC. Acute Mesenteric Ischemia; Diagnostic Approach and Surgical Treatment Semin Vasc Surg 2010; 23 (1): 9-20.

20. Kolkmann JJ, Groeneveld AB. Occlusive and non-occlusive gastrointestinal ischemia. A clinical review with specific emphasis on the diagnostic value of tonometry. Scand J Gastroenterol 1008; 225 (Suppl): 3.

21. Kanda T, Fujii H, Tani T et al. Intestinal fatty acid-binding protein is a useful diagnostic marker for mesenteric infarction in humus. Gastroenterology 1996; 110: 339.

22. Acosta S, Nilsson TK, Bjorck M. Preliminary study of D-dimer as a possible marker of acute bowel ischaemia. Br J Surg 2001; 88: 385-388.

23. Acosta S, Nilsson TK, Bjorck M. D-dimer testing in patients with suspected acute thromboembolic occlusion of the superior mesenteric artery. Br J Surg 2004; 91: 991-994.

24. Smerud MJ, Johnson CD, Stephens DH. Diagnosis of bowel infarction: a comparison of plain films and CT scans in 23 cases. Am J Roentgenol 1990; 154: 990.

25. Lee R, Tung HK, Tung PH et al. CT in acute mesenteric ischemia. Clin Radiol 2003; 58: 279.

26. Fleischmann D. Multiple detector-row $\mathrm{CT}$ angiography of the renal and mesenteric vessels. Eur J Radiol 2003; 45 (Suppl 1): S79-S87.

27. Horton KM, Fishman EK. Multidetector CT angiography in the diagnosis of mesenteric ischemia. Radiol Clin North Am 2007; 45: 275-288.

28. Savassi-Rocha PR, Veloso LF. Treatment of superior mesenteric artery embolism with a fibrinolytic agent: Case report and literature review. Hepatogastroenterology 2002; 49: 1307-1310.

29. Brountzos EN, Critselis A, Magoulas D, Kagianni E, Kelekis DA. Emergency endovascular treatment of a superior mesenteric artery occlusion. Cardiovasc Intervent Radiol 2001; 24: 57-60.

30. Bjorck M, Acosta S, Lindberg F, Troeng T, Bergqvist D. Revascularization of the superior mesenteric artery after acute thromboembolic occlusion. Br J Surg 2002; 89: 923-927. 
31. Carlos RC, Stanley JC, Stafford-Johnson D, Prince MR. Interobserver variability in the evaluation of chronic mesenteric ischemia with gadolinium-enhanced MR angiography. Acad Radiol 2001; 8: 879-887.

32. Sauerland S, Agresta F, Bergamaschi R et al. Laparoscopy for abdominal emergencies: Evidence-based guidelines of the European Association for Endoscopic Surgery. Surg Endosc 2006; 20: 14-29.

33. Modrall JG, Sadjadi J, Joiner DR et al. Comparison of superficial femoral vein and saphenous vein as conduits for mesenteric arterial bypass. J Vasc Surg 2003; 37: 362-366.

34. Kazmers A. Operative management of acute mesenteric ischemia. Ann Vasc Surg 1998; 12: 187-197.

35. Resch TA, Acosta S, Sonesson B. Endovascular Techniques in Acute Arterial Mesenteric Ischemia. Semin Vasc Surg 2010; 23 (1): 29-35.

36. Lim RP, Dowling RJ, Mitchell PJ, Vrazas JI, Thomson KR, Tress BM. Endovascular treatment of arterial mesenteric ischaemia: A retrospective review. Australas Radiol 2005; 49: 467-475.

37. Demirpolat G, Oran I, Tsmael TS, Parildar M, Memis A. Acute mesenteric ischemia: Endovascular therapy. Abdom Imaging 2007; 32: 299-303.

38. Hladik P, Raupach J, Lojik M et al. Treatment of acute mesenteric thrombosis/ischemia by transcatheter thromboaspiration. Surgery 2005; 137: 122-123.

39. Schoots IG, Levi MM, Reekers JA, Lameris JS, van Gulik TM. Thrombolytic therapy for acute superior mesenteric artery occlusion. J Vasc Interv Radiol 2005; 16: 317-329.

40. Gartenschlaeger S, Bender S, Maeurer J, Schroeder RJ. Successful percutaneous transluminal angioplasty and stenting in acute mesenteric ischemia. Cardiovasc Intervent Radiol 2008; 31: 398-400.

41. Chienne J. Complete obliteration of celiac and mesenteric arteries. J Anat Physiol 1869; 3: 63-72.

42. Shaw RS, Maynard EP. Acute and chronic thrombosis of the mesenteric arteries associated with malabsorption: A report of two cases successfully treated with thromboendarterectomy. N Engl J Med 1958; 258: 874-878.

43. Endean ED, Barnes SL, Kwolek CJ, Minion DJ, Schwarcz TH, Mentzer Jr. RM. Surgical management of thrombotic acute intestinal ischemia. Ann Surg 2001; 233: 801-808.

44. Thomas JH, Blake K, Pierce GE, Hermreck AS, Seigel E. The clinical course of asymptomatic mesenteric arterial stenosis. J Vasc Surg 1998; 27: $840-844$.

45. Mitchell EL, Moneta GL. Mesenteric duplex scanning. Perspect Vasc Surg Endovasc Ther 2006; 18: 175-183.

46. Schermerhorn ML, Giles KA, Hamdan AD, Wyers MC, Pomposelli FB. Mesenteric revascularization: Management and outcomes in the United States, 1988-2006. J Vasc Surg 2009; 50: 341-348.

47. Rosenblum JD, Boyle CM, Schwartz LB. The mesenteric circulation: Anatomy and physiology. Surg Clin N Am 1977; 77: 289-306.

48. Moneta GL, Taylor DC, Helton WS et al. Duplex ultrasound measurement of postprandial intestinal blood flow: Effect of meal composition. Gastroenterology 1988; 95: 1294.

49. Huber TS, Lee WA, Seeger JM. Chronic mesenteric ischemia. In: R.B. Rutherford, Editor, Vascular Surgery. WB Saunders, Philadelphia, PA (2005): 1732-1747.

50. Chandra A, Quinones-Baldrich WJ. Chronic Mesenteric Ischemia: How to Select Patients for Invasive Treatment. Semin Vasc Surg 2010; 23 (1): 21-28.
51. Park WM, Gloviczki P, Cherry KJ et al. Contemporary management of acute mesenteric ischemia: Factors associated with survival. J Vasc Surg 2002; 35: 445-552.

52. Oderich GS, Gloviczki P, Bower TC. Open Surgical Treatment for Chronic Mesenteric Ischemia in the Endovascular Era; When It is Necessary and what is the Preferred Technique? Semin Vasc Surg 2010; 23 (1): $36-46$.

53. Oderich GS, Bower TC, Sullivan TM, Bjarnason H, Cha S, Gloviczki P. Open versus endovascular revascularization for chronic mesenteric ischemia: Risk-stratified outcomes. J Vasc Surg 2009; 49: 1472-1479.

54. Park WM, Cherry Jr KJ, Chua HK et al. Current results of open revascularization for chronic mesenteric ischemia; A standard for comparison. J Vasc Surg 2002; 35: 853-859.

55. Mell MW, Acher CW, Hoch JR, Tefera G, Turnipseed WD. Outcomes after endarterectomy for chronic mesenteric ischemia. J Vasc Surg 2008; 48: 1132-1138.

56. Cho JS, Carr JA, Jacobsen G, Shepard AD, Nypaver TJ, Reddy DJ. Long-term outcome after mesenteric artery reconstruction: A 37-year experience. J Vasc Surg 2002; 35: 453-460.

57. Atkins MD, Kwolek CJ, LaMuraglia GM, Brewster DC, Chung TK, Cambria RP. Surgical revascularization versus endovascular therapy for chronic mesenteric ischemia; A comparative experience. J Vasc Surg 2007; 45: 1162-1171.

58. Oderich GS, Malgor R, Ricotta II JJ. Open and endovascular revascularization for chronic mesenteric ischemia: A tabular review of the literature. Ann Vasc Surg 2009; 23: 700-712.

59. Folky MI, Moneta GL, Abou-Zalam JrAM et al. Revascularization of the superior mesenteric artery aloe for treatment of intestinal ischemia. J Vasc Surg 2000; 32: 37-47.

60. Park WM, Cherry Jr KJ, Chua HK et al. Current results of open revascularization for chronic mesenteric ischemia: A standard for comparison. J Vasc Surg 2002; 35: 853-859.

61. Oderich GS, Macedo TA, Malgor R et al. Natural history, clinical and anatomical predictors of mesenteric artery stent restenosis in patients with chronic mesenteric ischemia, J Vasc Surg (2009) (supplement Annual Meeting of the Society for Vascular Surgery 2009).

62. Oderich GS, Macedo TA, Malgor R et al. Anatomic measurements and factors associated with embolic events during superior mesenteric artery stenting: Implications for use of embolic protection devices. Presented at the 2009 Society for Clinical Vascular Surgery Annual Meeting, Fort Lauderdale, FL, 2009.

63. Schermerhorn ML, Giles KA, Hamdan AD, Wyers MC, Pomposelli FB. Mesenteric revascularization: Management and outcomes in the United States, 1988-2006. J Vasc Surg 2009; 50: 341-348.

64. Brown DJ, Schermerhorn ML, Powell RJ et al. Mesenteric stenting for chronic mesenteric ischemia. J Vasc Surg 2005; 42: 268-274.

65. Chahid T, Alfidja AT, Biard M, Ravel A, Garcier JM, Boyer L. Endovascular treatment of chronic mesenteric ischemia: Results in 14 patients. Cardiovasc Intervent Radiol 2004; 27: 637-642.

66. Björck M, Wanhainen A. Nonocclusive Mesenteric Hypoperfusion Syndromes: Recognition and Treatment. Semin Vasc Surg 2010; 23 (1): $54-64$.

Received February 9, 2011. Accepted December 17, 2012. 